\title{
Restaging: Should We PERCIST Without Pattern Recognition?
}

$\mathbf{O}$ ne of the great capacities of the human brain is its ability to recognize patterns. We can instantly identify a friend we haven't seen for years, even though he or she may have put on weight, lost hair, or gathered a few wrinkles. Pattern recognition is equally pivotal in the interpretation of imaging studies. In the case of PET, the distribution and intensity of radiotracer uptake, considered in the context of the clinical history, help to suggest a diagnosis. Like encountering an old friend, the experienced physician can formulate an impression of a PET scan within seconds of viewing the maximum-intensity-projection image, finding order within a mass of data. This global Gestalt approach to imaging plays an invaluable role that surpasses even a careful step-by-step

\section{See page 1833}

algorithmic approach $(1,2)$. Nevertheless, because PET enables quantification of ${ }^{18} \mathrm{~F}-\mathrm{FDG}$ uptake, correlating with the rate of glycolysis, there is a temptation to use this capability for image interpretation (e.g., using semiquantitative measures such as the standardized uptake value [SUV]). The value of pattern recognition has, at times, been forgotten, as evidenced by the adoption of an arbitrary cutoff SUV of 2.5 for characterization of pulmonary nodules (3). Given the range of pro-

Received Jun. 28, 2010; revision accepted Jul. 2, 2010.

For correspondence or reprints contact: Rodney Hicks, Centre for Cancer Imaging, Peter MacCallum Cancer Centre, 12 St. Andrew's PI., East Melbourne, Victoria 3002, Australia. E-mail: Rod.Hicks@petermac.org

COPYRIGHT (C) 2010 by the Society of Nuclear Medicine, Inc.

DOI: 10.2967/jnumed.110.079293 cesses that can augment glucose use in the lungs, it is hardly surprising that this disuse of pattern recognition can lead to inaccuracies (4).

Just as seeing a familiar face in an unfamiliar environment can cause uncertainty, unexpected foci of uptake attract our attention but demand caution. Better localization and characterization of anatomic features with PET/ CT has substantially improved accuracy (5). Even so, many clinicians do not appreciate the irony of using pattern recognition to interpret the $\mathrm{CT}$ component while relying on arbitrary cutoffs for the intensity of ${ }^{18} \mathrm{~F}-\mathrm{FDG}$ uptake. Many potential pitfalls in the interpretation of PET/CT can be avoided by learning patterns associated with benign processes. Symmetric uptake in hilar nodes is a well-known pattern for differentiating inflammatory from malignant lymphadenopathy (6). The typical distribution of brown fat $(7,8)$, the pattern of intense ${ }^{18} \mathrm{~F}-\mathrm{FDG}$ uptake in the bowel of patients treated with metformin (9), or elastofibroma dorsi (10) are surprisingly easy to recognize. Indeed, once you appreciate such a pattern, you wonder how you missed it despite seeing it many times before.

Relying on intensity of uptake alone is like relying only on the color of an individual's hair to recognize him or her, particularly when hair dyes can alter this characteristic. It is equally counterproductive to rely solely on the intensity of ${ }^{18} \mathrm{~F}-\mathrm{FDG}$ uptake for response assessment when a component of inflammation should be anticipated, as it is for localized treatments such as surgery, radiotherapy, or radiofrequency ablation (RFA). These also result in distorted anatomy, limiting the value of anatomic response criteria based on size such as the Response Evaluation Criteria in
Solid Tumors and its latest iteration (11). These considerations are highlighted by the study of Singnurkar et al. in the current issue of The Journal of Nuclear Medicine, which investigates the role of ${ }^{18} \mathrm{~F}-\mathrm{FDG} \mathrm{PET} / \mathrm{CT}$ for assessment of local recurrence after RFA of malignant lung lesions (12).

The technique of RFA involves inserting a specialized needle into the lesion and using an alternating current to generate energy that results in thermal coagulation necrosis of the lesion and surrounding pulmonary parenchyma. Evaluation of treatment efficacy based on morphologic criteria is compromised because RFA results in a mass that is almost always larger than the original lesion, even after successful ablation. Nevertheless, early detection of recurrence is important, because repeated RFA can be performed and is best performed on small recurrences. The question raised by the current study is whether ${ }^{18} \mathrm{~F}$ FDG PET could identify residual disease more robustly than CT.

There is no doubt that imaging metabolic pathways such as glycolysis with ${ }^{18} \mathrm{~F}$-FDG PET provides additional information for response assessment. This is particularly obvious with interval development of distant disease or loss of metabolic activity in the residual mass (13). The advantages of PET are, for example, clear in several targeted therapies such as imatinib for gastrointestinal stromal tumors (14), for which there is a rapid reduction in metabolic activity as early as $24 \mathrm{~h}$ of initiation of therapy (15). In these contexts, PET is vastly superior to anatomic restaging. However, the challenge occurs when ${ }^{18} \mathrm{~F}-\mathrm{FDG}$ uptake is still present or even increased.

A framework titled PET Response Criteria in Solid Tumors (PERCIST) 
has been proposed to overcome the limitations of anatomic response using the ability of PET to quantify the degree of reduction in ${ }^{18} \mathrm{~F}-\mathrm{FDG}(11)$. These criteria are based on the premise that metabolic response is a continuous and time-dependent variable. An SUV decrease of $30 \%$ or greater is defined as a medically relevant beneficial change or response, although the rationale for this choice seems to be based primarily on the presumption that such change is unlikely to be related to technical factors. A complete metabolic response requires the complete resolution of uptake so that it is less than liver activity and indistinguishable from surrounding background blood-pool levels. The framework has the advantage of being easily applied, with high reproducibility, making it particularly useful for hypothesis testing in the context of a clinical trial. Furthermore, it can be generalized to a wide variety of malignancies and situations and avoids the conceptual limitations associated with defining an optimal SUV threshold based on post hoc analysis of data.

However, as we have alluded, there are limitations in using the intensity of uptake alone to assess response, particularly when inflammatory changes within the tumor and surrounding tissue are an invariable consequence of the treatment. Inflammatory change can be florid, resulting in high-intensity metabolic abnormality, making SUV analysis of limited use. A change in the pattern of uptake, however, can be highly specific and predictable, depending on the type of therapy used. In general, focal uptake, when combined with a round or ovoid mass on CT, is a key feature of malignant uptake. Linear or geographic uptake not respecting anatomic boundaries, on the other hand, is more often a feature of inflammatory etiologies.

Exemplifying these principles, Singnurkar et al. performed PET/CT at 1-4 mo after RFA of pulmonary malignancies and dichotomized patients qualitatively using the pattern of uptake (12). The authors defined 6 patterns of ${ }^{18} \mathrm{~F}$-FDG uptake and divided these into favorable and unfavorable categories based on posttreatment inflammation that did not generally appear as focal uptake but rather as a rim of mild heterogeneous uptake. Local recurrences were observed in 10 of 12 patients with unfavorable uptake, compared with 9 of 29 patients with a favorable uptake pattern. In contrast, the change in SUV between pre- and postablation scans varied widely and was not predictive of recurrence-free survival.

Our group has demonstrated the utility of ${ }^{18}$ F-FDG PET treatment response soon after radical thoracic radiotherapy for non-small cell lung carcinoma (16). A pattern-recognition algorithm comparing pre- and posttreatment scans was used, integrating the expectation of radiation-induced inflammatory uptake. Increased ${ }^{18} \mathrm{~F}$ FDG uptake in a geographic distribution in normal lung or pleura was associated with a greater likelihood of response within the primary mass. Thus, the presence of increasing intensity of ${ }^{18} \mathrm{~F}-\mathrm{FDG}$ activity on the posttreatment scan in a defined pattern did not hamper interpretation as a purely quantitative approach would-it actually enhanced response assessment. Other studies have shown similar advantages to using pattern characterization for the restaging of treatment. Using 6 predefined PET/CT patterns for the diagnosis of surgical staple line recurrence in patients with colorectal carcinoma, Shyn et al. demonstrated a sensitivity and specificity of $100 \%$ and $97.1 \%$, respectively (17). The high sensitivity and specificity were contrasted by a lower accuracy using maximum SUV, even when defining optimal cutoff values using post hoc analysis of data. Our group has further demonstrated the utility of pattern recognition in restaging rectal carcinoma after chemoradiotherapy (18). A graded schema was used to integrate changes in skeletal muscle and bowel within the radiation field. Integrating these patterns allowed the correct reclassification of patients labeled as partial metabolic responders to complete metabolic responders.
Pattern recognition is a core component of cancer imaging but is frequently overlooked in the research arena, in which there is a bias toward performing statistical analysis of quantitative data. The qualitative nature of pattern recognition produces data that are seemingly less scientific in that the data are subjective rather than objective. Nevertheless, our brains are highly tuned to detecting and making sense of patterns and adapt rapidly by learning new patterns. It is refreshing to see the research by Singnurkar et al. incorporating the reader's evaluation of a change in pattern of uptake rather than concentrating on quantitative variables alone. We need to burn their description of unfavorable patterns of ${ }^{18} \mathrm{~F}-$ FDG uptake into our brains rather than always PERCISTing with SUV criteria of response. In the future, the application of computer intelligence to quantify changes in tissue distribution and artificial intelligence coded by experienced readers may provide the benefits of both approaches.

\section{Michael S. Hofman \\ Peter MacCallum Cancer Centre}

East Melbourne, Australia

Rodney J. Hicks

University of Melbourne

Melbourne, Australia

\section{REFERENCES}

1. Koontz NA, Gunderman RB. Gestalt theory: implications for radiology education. AJR. 2008; 190:1156-1160.

2. Oestmann JW, Greene R, Bourgouin PM, Linetsky L, Llewellyn HJ. Chest "gestalt" and detectability of lung lesions. Eur J Radiol. 1993; 16:154-157.

3. Lowe VJ, Hoffman JM, DeLong DM, Patz EF, Coleman RE. Semiquantitative and visual analysis of FDG-PET images in pulmonary abnormalities. J Nucl Med. 1994;35:1771-1776.

4. Hashimoto Y, Tsujikawa T, Kondo C, et al. Accuracy of PET for diagnosis of solid pulmonary lesions with ${ }^{18} \mathrm{~F}$-FDG uptake below the standardized uptake value of 2.5. J Nucl Med. 2006;47:426-431.

5. Metser U, Miller E, Lerman H, Even-Sapir E. Benign nonphysiologic lesions with increased ${ }^{18}$ F-FDG uptake on PET/CT: characterization and incidence. AJR. 2007;189:1203-1210.

6. Karam M, Roberts-Klein S, Shet N, Chang J, Feustel P. Bilateral hilar foci on ${ }^{18}$ F-FDG PET scan in patients without lung cancer: variables associated with benign and malignant etiology. J Nucl Med. 2008;49:1429-1436. 
7. Hany TF, Gharehpapagh E, Kamel EM, Buck A, Himms-Hagen J, von Schulthess GK. Brown adipose tissue: a factor to consider in symmetrical tracer uptake in the neck and upper chest region. Eur J Nucl Med Mol Imaging. 2002;29:1393-1398.

8. Cohade C, Osman M, Pannu HK, Wahl RL. Uptake in supraclavicular area fat ("USA-Fat"): description on ${ }^{18}$ F-FDG PET/CT. J Nucl Med. 2003;44:170-176.

9. Gontier E, Fourme E, Wartski M, et al. High and typical ${ }^{18}$ F-FDG bowel uptake in patients treated with metformin. Eur J Nucl Med Mol Imaging. 2008;35:95-99.

10. Patrikeos A, Breidahl W, Robins P. F-18 FDG uptake associated with Elastofibroma dorsi. Clin Nucl Med. 2005;30:617-618.

11. Wahl RL, Jacene H, Kasamon Y, Lodge MA. From RECIST to PERCIST: evolving considerations for PET response criteria in solid tumors. $\mathrm{J} \mathrm{Nucl} \mathrm{Med}$. 2009;50(suppl 1):122S-150S.
12. Singnurkar A, Solomon S, Gönen M, Larson SM, Schöder H. ${ }^{18}$ F-FDG PET/CT for the prediction and detection of local recurrence after radiofrequency ablation of malignant lung lesions. J Nucl Med. 2010;51:1833-1840.

13. Jerusalem G, Beguin Y, Fassotte MF, et al. Wholebody positron emission tomography using ${ }^{18} \mathrm{~F}$ fluorodeoxyglucose for posttreatment evaluation in Hodgkin's disease and non-Hodgkin's lymphoma has higher diagnostic and prognostic value than classical computed tomography scan imaging. Blood. 1999;94:429-433.

14. McAuliffe JC, Hunt KK, Lazar AJ, et al. A randomized, phase II study of preoperative plus postoperative imatinib in GIST: evidence of rapid radiographic response and temporal induction of tumor cell apoptosis. Ann Surg Oncol. 2009;16: 910-919.

15. Cullinane C, Dorow DS, Kansara M, et al. An in vivo tumor model exploiting metabolic response as a biomarker for targeted drug development. Cancer Res. 2005;65:9633-9636.

16. Hicks RJ, Mac Manus MP, Matthews JP, et al. Early FDG-PET imaging after radical radiotherapy for non-small-cell lung cancer: inflammatory changes in normal tissues correlate with tumor response and do not confound therapeutic response evaluation. Int J Radiat Oncol Biol Phys. 2004;60: $412-418$

17. Shyn PB, Madan R, Wu C, Erturk SM, Silverman SG. PET/CT pattern analysis for surgical staple line recurrence in patients with colorectal cancer. AJR. 2010;194:414-421.

18. Kalff V, Ware R, Heriot A, Chao M, Drummond E, Hicks RJ. Radiation changes do not interfere with postchemoradiation restaging of patients with rectal cancer by FDG PET/CT before curative surgical therapy. Int $J$ Radiat Oncol Biol Phys. 2009;74:60-66. 